\title{
Molecular profiling in the treatment of colorectal cancer: focus on regorafenib
}

This article was published in the following Dove Press journal:

OncoTargets and Therapy

15 October 2015

Number of times this article has been viewed

Yiyi Yan

Axel Grothey

Department of Medical Oncology, Mayo Clinic, Rochester, MN, USA
Correspondence: Axel Grothey

Department of Medical Oncology, Mayo

Clinic, 200 First Street SW, Rochester,

MN 55905, USA

Tel +I 50728425 II

Email grothey.axel@mayo.edu

\begin{abstract}
Metastatic colorectal cancer $(\mathrm{mCRC})$ is a highly heterogeneous disease. Its treatment outcome has been significantly improved over the last decade with the incorporation of biological targeted therapies, including anti-EGFR antibodies, cetuximab and panitumumab, and VEGF inhibitors, bevacizumab, ramucirumab, and aflibercept. The identification of predictive biomarkers has further improved the survival by accurately selecting patients who are most likely to benefit from these treatments, such as $R A S$ mutation profiling for EGFR antibodies. Regorafenib is a multikinase inhibitor currently used as late line therapy for mCRC. The molecular and genetic markers associated with regorafenib treatment response are yet to be characterized. Here, we review currently available clinical evidence of mCRC molecular profiling, such as $R A S, B R A F$, and MMR testing, and its role in targeted therapies with special focus on regorafenib treatment.
\end{abstract}

Keywords: metastatic colon cancer, targeted therapy, molecular profiling, regorafenib

\section{Introduction}

Metastatic colorectal cancer (mCRC) is a significant cause of mortality and morbidity in the US. In 2015 alone, an estimated 132,700 newly diagnosed cases of CRC and an estimated 49,700 deaths from CRC were expected. Approximately $25 \%-30 \%$ of patients with newly diagnosed CRC have evidence of metastases upon diagnosis. ${ }^{1}$ While some patients with $\mathrm{mCRC}$ with limited metastatic spread can be rendered free of disease long-term by a multidisciplinary approach, for the majority of the patients, treatment generally consists of medical therapy with palliative intent. Since the 1980s, 5-fluorouracil based chemotherapy has been the backbone of therapy, with the incorporation of oxaliplatin and irinotecan in the 1990s. Over the past decade, the addition of targeted biologic agents, such as antibodies against the EGFR monoclonal antibodies (mAbs), cetuximab, panitumumab, and inhibitors of the VEGF system like bevacizumab and aflibercept, to combination chemotherapy has changed the landscape of mCRC treatment, with a doubling of the median overall survival (OS) and improved long-term survival. Regorafenib, an oral multikinase inhibitor, was later approved in 2012, providing further treatment options for mCRC patients who failed multiple lines of therapy.

Despite the promising outcome of targeted therapies in $\mathrm{mCRC}$, primary and secondary resistance to therapy remains a clinical challenge in $\mathrm{mCRC}$. As we advance our knowledge in mCRC tumor biology and genetics, it is of special importance to understand the underlying molecular profiles in relationship to targeted therapy. This allows the development of reliable biomarkers to more accurately select patients who will have greatest benefit from such therapy. Although multiple molecular makers have been well established to guide some targeted therapy for $\mathrm{mCRC}$, exemplified by 
RAS testing for EGFR mAbs, there is no validated molecular testing to improve the outcome with regorafenib treatment.

In this review, we summarize existing evidence of the value of molecular profiling in $\mathrm{mCRC}$ for targeted therapies with special focus on the currently available clinical evidence on molecular biomarkers associated with regorafenib treatment.

\section{Overview of current biomarkers in CRC treatment RAS analysis and anti-EGFR treatment in $\mathrm{mCRC}$}

$R A S$ encodes a family of small GTP-binding proteins that act as self-inactivating signal transducers in response to stimulation of a cell surface receptor, including EGFR. Oncogenic mutations of KRAS are found in approximately $40 \%$ of mCRC tumors. It results in constitutive activation of the RAS/ RAF/ERK pathway, rendering EGFR inhibitor ineffective. ${ }^{2}$ $K R A S$ and $N R A S$ are closely related $R A S$ oncogene family members, and CRCs can harbor mutations in either gene, which tend to be mutually exclusive, suggesting functional redundancy. ${ }^{3}$ Resistance to anti-EGFR therapies can also be mediated by any activating mutation in exons 2,3 , and 4 of KRAS and NRAS. ${ }^{4-6}$

Multiple recent studies have established the role of RAS analysis in identifying patients who are likely to respond to anti-EGFR agents.

Panitumumab is a fully human $\mathrm{mAb}$ for EGFR extracellular domain. Its benefit as monotherapy in chemotherapy-refractory mCRC was demonstrated in the 408 trial, comparing panitumumab with best supportive care (BSC) to BSC alone in patients unselected for $K R A S$ status. The panitumumab treated population had improved median progression-free survival (PFS) (8 weeks vs 7.3 weeks, hazard ratio [HR], 0.54, 95\% confidence interval [CI], 0.44 to $0.66, P<0.0001){ }^{7}$ Post hoc analysis based on the $K R A S$ status (exon 2 with codon 12 and codon 13) was later carried out based on the previous observations that mutant $K R A S$ might correlate with poor prognosis in mCRC and other types of tumors. ${ }^{8,9}$ This reanalysis showed that the benefit of panitumumab was limited to patients with $K R A S$ wild-type (wt) CRC. ${ }^{10}$

Extended $R A S$ analysis was also performed on 408 trial data. In $K R A S$ wt patients, effect of panitumumab treatment on PFS was studied on multiple genotypes including NRAS, BRAF, PIK3CA, AKT, TP53, and CTNNB1. A favorable PFS benefit with panitumumab treatment was observed among those with wt NRAS (HR, 0.39; 95\% CI, 0.27-0.56) and wt BRAF (HR, 0.37; 95\% CI, 0.24-0.55), but not mutant NRAS (HR, 1.94; 95\% CI, 0.44-8.44, P=0.379). ${ }^{4}$

The detrimental impact of panitumumab treatment in patients with $R A S$ mutation beyond $K R A S$ exon 2 was observed in multiple studies.

For example, in the PRIME trial, 5,6 the association of $R A S$ mutations beyond $K R A S$ exon 2 and anti-EGFR treatment efficacy was assessed in patients treated with panitumumab plus FOLFOX4 vs FOLFOX4 alone. Tumors were analyzed for full spectrum of $R A S$ mutations (KRAS and $N R A S$ exon $2,3,4)$ as well as $B R A F$ V600E mutation. In patients without any RAS mutations, panitumumab plus FOLFOX4 was associated with a significant improvement in PFS and OS as compared to FOLFOX4 alone (median PFS 10.1 vs 7.9 months, $P=0.004$; median OS 26.0 vs 20.2 months, $P=0.04)$. In the subgroup of patients carrying $R A S$ mutations other than KRAS exon 2, shorter PFS and OS associated with panitumumab combination treatment than with FOLFOX4 alone was shown, consistent with the outcome observed in patients with $K R A S$ exon 2 mutated tumors. These results confirmed the role of $R A S$ mutations beyond $K R A S$ exon 2 as predictive markers for an adverse outcome for panitumumab treatment, suggesting the importance of extended $R A S$ testing to provide the greatest treatment benefit with panitumumab.

Another anti-EGFR agent, cetuximab, an IgG1 chimeric monoclonal EGFR antibody was also extensively studied in mCRC treatment. It binds to the EGFR, competitively inhibiting ligand binding and inducing receptor dimerization and internalization. The efficacy of cetuximab vs panitumumab was compared in KRAS wt chemotherapy-refractory patients in the ASPECCT trial, a non-inferiority Phase 3 study. ${ }^{11}$ Panitumumab was demonstrated to be non-inferior to cetuximab, with a median OS of 10.0 months vs 10.4 months, respectively (HR, 0.97; 95\% CI, 0.84-1.11).

The efficacy of cetuximab compared to BSC in patients with metastatic CRC was assessed in the NCIC CO.17 trial. Cetuximab improved OS and PFS in patients with detectable EGFR regardless of KRAS status. ${ }^{12}$ Benefit in OS and PFS with cetuximab treatment was significantly greater in patients with wt KRAS (exon 2, codons 12/13) (median OS 9.5 vs 4.8 months; HR, 0.55; 95\% CI, 0.41-0.74; median PFS 3.7 months vs 1.9 months; HR, 0.40; 95\% CI, 0.30-0.54, $P<0.001)$. However, the OS in patients receiving BSC was not affected by $K R A S$ mutation status. ${ }^{13}$

In the CRYSTAL trial, the efficacy of cetuximab treatment in combination with FOLFIRI vs FOLFIRI alone as first-line therapy in $\mathrm{mCRC}$ was investigated. This trial 
demonstrated the benefit of cetuximab in PFS, OS, and tumor response, and these benefits were limited to KRAS wt patients. ${ }^{14,15}$

Taken together, these clinical trials demonstrated the importance of extended $R A S$ mutation analysis, rather than just in $K R A S$ exon 2, in optimal patient selection to benefit from anti-EGFR therapy. According to current guidelines, ${ }^{16}$ comprehensive mutation testing in KRAS and NRAS exon 2, 3 , and 4 is mandated for consideration of anti-EGFR therapy; cetuximab and panitumumab should be avoided for patients with any $R A S$ mutations.

\section{BRAF mutations and RAF/MEK inhibitor treatment in CRC}

Although extended $R A S$ testing allows identification of appropriate patients to benefit from anti-EGFR treatment, a significant subset of patients with wt $R A S$ fail to show improved outcome from such a therapy. Therefore, recognition of other biomarkers beyond $R A S$ would optimize the outcome of personalized treatment.

In addition to $R A S$, activating mutations in $B R A F$, predominantly V600E, are among one of the first events in colorectal carcinogenesis, and are identified in $8 \%-10 \%$ of CRC patients. They are considered as mutually exclusive to $R A S$ mutations. ${ }^{17} B R A F$ mutant tumors are associated with typical clinical characteristics, including right-sided, high-grade mucinous histology, high frequency of lymph node and peritoneal metastasis and microsatellite instability (MSI), with distinctive gene expression patterns. ${ }^{18,19}$ A correlation between $B R A F \mathrm{~V} 600 \mathrm{E}$ mutation and poor prognosis and aggressive phenotype has been well demonstrated. ${ }^{15,20,21}$ Given the aggressiveness of $B R A F$ mutated CRC, intensification of first-line chemotherapy has shown outcome benefit. FOLFOXIRI plus bevacizumab have been shown to improve PFS and OS in BRAF mutated mCRC as compared to FOLFIRI plus bevacizumab, justifying the intensive upfront approach in this subgroup of patients with an unfavorable prognosis..$^{22,23}$

The predictive value of $B R A F$ mutation status in outcomes of anti-EGFR treatment was also assessed in multiple clinical studies and meta-analyses. In $K R A S$ wt patients, favorable effects of anti-EGFR mAbs on OS, PFS, and overall response rate (ORR) were observed in patients with wt $B R A F$ CRC compared to $B R A F$ mutants. ${ }^{4,5,24}$ Additionally, according to a recent meta-analysis examining the impact of cetuximab and panitumumab in $B R A F$-mutated $\mathrm{mCRC}$ patients, there is no overall benefit (OS, PFS, and ORR) with anti-EGFR treatment in patients with $B R A F$ mutated tumors. ${ }^{25}$
Unlike in melanoma, treatment with BRAF inhibitor failed to provide benefit in $B R A F$ mutated mCRC. ${ }^{26}$ Treatment resistance of CRC to BRAF inhibitor, vemurafenib, was found to be caused by feedback activation of EGFR, mediated through the MAPK/ERK pathway. In vivo and in vitro studies demonstrated synergistic activity of vemurafenib and EGFR inhibitor in BRAF mutated CRC. ${ }^{27,28}$ In addition, hyperactivation of PI3K/Akt/PTEN pathway can also result in BRAF inhibition resistance. ${ }^{29,30}$ These preclinical studies provided rationale supporting combination target therapies in BRAF mutated CRC (Table 1).

Combination regimens including a BRAF inhibitor have been investigated for the treatment of $B R A F$ mutant $C R C$ in multiple recent clinical trials. Dabrafenib, a BRAF inhibitor, combined with the MEK inhibitor, trametinib, showed low activity in ORR and PFS. ${ }^{31}$ Combination of BRAF inhibitor and anti-EGFR mAbs, panitumumab or cetuximab, was also examined by different groups. In a recent pilot clinical trial, ${ }^{32}$ vemurafenib plus panitumumab treatment was able to provide modest clinical benefit. Similar clinical outcome was also demonstrated when cetuximab was combined with vemurafenib. ${ }^{33}$

Based on the in vitro evidence that combined EGFR and MEK inhibitors synergize to inhibit BRAF mutated CRC, ${ }^{27}$ dabrafenib plus panitumumab was evaluated with or without trametinib. ${ }^{34}$ Triplet treatment demonstrated a response rate (RR) of $26 \%$ compared to $10 \%$ in the dabrafenib plus panitumumab group with tolerable toxicity profile. ${ }^{34}$ Given the important role of PI3K/Akt/PTEN pathway in the BRAF inhibitor treatment resistance, a specific PI3K inhibitor, alpelisib, was studied in combination with encorafenib,

Table I Studies of BRAF-targeted therapies in mCRC

\begin{tabular}{|c|c|c|c|}
\hline $\begin{array}{l}\text { Studies } \\
\text { (Author) }\end{array}$ & Treatment & ORR & $\begin{array}{l}\text { PFS } \\
\text { (months) }\end{array}$ \\
\hline Kopetz et $\mathrm{al}^{26}$ & Vemurafenib & $5 \%$ & 2.1 \\
\hline Falchook et $\mathrm{al}^{78}$ & Dabrafenib & $10 \%$ & NR \\
\hline Corcoran et $\mathrm{al}^{31}$ & Dabrafenib + trametinib & $12 \%$ & 3.5 \\
\hline Yaeger et $\mathrm{al}^{32}$ & Vemurafenib + panitumumab & $13 \%$ & 3.2 \\
\hline Tabernero et $\mathrm{al}^{33}$ & Vemurafenib + cetuximab & $20 \%$ & 3.2 \\
\hline Atreya et $\mathrm{al}^{34}$ & Dabrafenib + panitumumab & $10 \%$ & 3.4 \\
\hline Atreya et $\mathrm{al}^{34}$ & $\begin{array}{l}\text { Dabrafenib + panitumumab }+ \\
\text { trametinib }\end{array}$ & $26 \%$ & 4.1 \\
\hline Tabernero et $\mathrm{al}^{35}$ & Encorafenib + cetuximab & $23 \%$ & 4.0 \\
\hline Tabernero et $\mathrm{al}^{35}$ & $\begin{array}{l}\text { Encorafenib + cetuximab + } \\
\text { alpelisib }\end{array}$ & $32 \%$ & 4.4 \\
\hline Hong et $\mathrm{al}^{36}$ & $\begin{array}{l}\text { Vemurafenib + cetuximab }+ \\
\text { irinotecan }\end{array}$ & $35 \%$ & 7.7 \\
\hline
\end{tabular}

Abbreviations: $\mathrm{mCRC}$, metastatic colorectal cancer; ORR, overall response rate; PFS, progression-free survival; NR, not reported. 
a BRAF inhibitor, and cetuximab. Addition of alpelisib improved RR to $32 \%$ compared to $23 \%$ in encorafenib plus cetuximab only group. ${ }^{35}$ A triplet regimen including standard chemotherapy resulted in remarkably long PFS (median PFS of 7.7 months) and a high RR of 35\% when irinotecan was added to vemurafenib plus cetuximab. ${ }^{36}$ These significant improvements in treatment of this aggressive subtype of mCRC substantiate the importance of further understanding and identification of biomarkers in CRC.

\section{MSI and PD-I blockade in mCRC}

Over the last decade, the understanding of the immune checkpoint pathways, such as the PD-1 pathway, has paved the way for immunotherapy in solid tumors.

The PD-1 receptor is expressed on the cell surface of activated T-cells under normal conditions. By binding to its ligand (PD-L1 and PD-L2), PD-1 downregulates T-cell activation and therefore dampens unwarranted and excessive immune responses, including autoimmunity. ${ }^{37,38}$ The interaction between PD-L1 expressed on tumor and stromal cells and PD-1 on T-cells can trigger inhibitory signaling pathways that reduce effector cell functions and T-cell-killing capacity, ${ }^{39}$ enabling tumor cells to evade immune surveillance. Blocking the PD-1/PD-L1 interaction by antibodies has led to remarkable antitumor response in different types of solid tumors, including non-small-cell lung cancer, renal cell carcinoma, and bladder cancer. ${ }^{40-45}$ PD-1 blockade also demonstrated activity towards $\mathrm{CRC}$, although with a low response rate compared to in other types of tumors. ${ }^{43,46}$ Success in PD-1 blockade has led to a search for molecular and genetic biomarkers that correlate to treatment response. Although an association was observed between high baseline PD-L1 expression and clinical response, patients with low/negative PD-L1 expression also benefit from PD-1 blockade. ${ }^{40}$ The presence of pretreatment tumor-infiltrating CD8 T-cells has later been shown to tightly correlate with anti-PD-1 response. ${ }^{47,48}$ However, this correlation lacks tumor specificity.

Deficiency in the expression of MMR enzymes can be seen in a small fraction of mCRC patients, which results in MSI and a higher somatic mutation burden than in MMR proficient mCRC. ${ }^{49}$ These mutations expressed by tumors can not only be exploited as a basis for targeted therapies, but can also stimulate anti-tumor immunogenicity through the expression of tumor specific antigens (neoantigens). Previous studies have shown that MSI is associated with more prominent lymphocyte infiltration and that tumors with higher somatic mutations are more responsive to immunotherapy. ${ }_{4}^{43,50}$
MMR status was recently demonstrated to be associated with a response to anti-PD-1 treatment in CRC. ${ }^{51,52}$ In a recent Phase 2 trial, clinically relevant activity was observed with pembrolizumab, a humanized antibody against PD-1 in MMR-deficient CRC, with immune-related ORR of $40 \%$ and immune-related PFS at 20 weeks of $78 \%$, respectively, compared to $0 \%$ and $11 \%$ for MMR-proficient CRCs. Response rate and disease control rates by "response evaluation criteria in solid tumors (RECIST)" criteria were 40\% and 90\% in MMR-deficient CRC, and $0 \%$ and 11\% in MMR-proficient CRC. ${ }^{52,53}$ This study demonstrated a role of MSI testing as a potential molecular biomarker to select CRC patients for individualized immune treatment.

\section{Molecular profiling and regorafenib Regorafenib in treatment of $\mathrm{mCRC}$}

Regorafenib is an oral multikinase inhibitor approved for the treatment of patients with $\mathrm{mCRC}$ who have previously been treated with fluoropyrimidine-, oxaliplatin-, and irinotecanbased chemotherapy; anti-VEGF therapy, and anti-EGFR agent if KRAS wt. ${ }^{54}$ It potently inhibits both intracellular and membrane-bound angiogenic and stromal RTKs that promote tumor neovascularization and vessel stabilization in the microenvironment, including VEGF receptor 1-3, PDGFR- $\beta$, TIE2, and FGF receptor 1 . It also inhibits oncogenic RTKs, such as RET, KIT, and wt and V600E mutated BRAF (Table 2). ${ }^{55}$ Anti-proliferative effects through RAF/ MEK/ERK signaling and anti-tumor activity were observed in in vitro assays in $\mathrm{CRC}$, breast cancer, and renal cell carcinoma mouse xenograft models. ${ }^{55}$ In the murine metastatic CRC model, regorafenib showed strong anti-angiogenic, anti-tumorigenic, and anti-metastatic effects, suggestive of its potential in the treatment of advanced CRCs. ${ }^{56}$

A Phase $1 / 1$ b study to assess the pharmacokinetics, toxicity, and response of regorafenib was conducted in 38 patients

Table 2 Biochemical activities of regorafenib in in vitro kinase assays

\begin{tabular}{ll}
\hline Kinase targets $^{55}$ & $\begin{array}{l}\text { Regorafenib IC } \\
\text { (nM) } \pm \text { SD }\end{array}$ \\
\hline VEGFRI & $13 \pm 0.4$ \\
TIE2 & $311 \pm 46$ \\
PDGFR- $\beta$ & $22 \pm 3$ \\
FGFRI & $202 \pm 18$ \\
KIT & $7 \pm 2$ \\
RET & $1.5 \pm 0.7$ \\
RAF-I & $2.5 \pm 0.6$ \\
B-RAF & $2.5 \pm 10$ \\
B-RAF & $19 \pm 6$ \\
\hline
\end{tabular}

Abbreviations: $I C_{50}$, inhibitory concentration of $50 \%$; SD, standard deviation. 
Table 3 Studies of single agent regorafenib in mCRC

\begin{tabular}{|c|c|c|c|c|}
\hline $\begin{array}{l}\text { Studies } \\
\text { (Author/Year) }\end{array}$ & Design & Treatment & Median PFS (months) & Median OS \\
\hline $\begin{array}{l}\text { Strumberg } \\
\text { et a }\left.\right|^{57}(2012)\end{array}$ & Phase I, non-randomized, n=38 & $\begin{array}{l}60-220 \mathrm{mg} \text { daily } \\
\text { (21 days on/7 days off) }\end{array}$ & $3.6(95 \% \mathrm{Cl}, 2.2-5.6)$ & NR \\
\hline $\begin{array}{l}\text { Grothey et al }{ }^{59} \\
(2013)\end{array}$ & $\begin{array}{l}\text { Phase 3, randomized } \\
\text { (regorafenib:placebo }=2: \mathrm{I}, \mathrm{n}=760 \text { ) }\end{array}$ & $\begin{array}{l}160 \mathrm{mg} \text { daily } \\
\text { (21 days on/7 days off) }\end{array}$ & $\begin{array}{l}\text { I.9 vs I.7 (PFS HR, 0.49; } \\
95 \% \mathrm{Cl}, 0.42-0.58 \mathrm{I}, \mathrm{P}<0.000 \mathrm{I})\end{array}$ & $\begin{array}{l}6.4 \text { vs } 5.0(\mathrm{HR}, 0.77 ; 95 \% \mathrm{Cl} \text {, } \\
0.64-0.94, P=0.0052)\end{array}$ \\
\hline Li et $a^{160}(2015)$ & $\begin{array}{l}\text { Phase 3, randomized } \\
\text { (regorafenib:placebo }=2: 1, n=204 \text { ) }\end{array}$ & $\begin{array}{l}160 \mathrm{mg} \text { daily } \\
\text { (21 days on/7 days off) }\end{array}$ & $\begin{array}{l}3.2 \text { vs I.7 (PFS HR, } 0.3 \mathrm{I} \text {; } \\
95 \% \mathrm{Cl}, 0.22-0.44, P<0.000 \mathrm{I})\end{array}$ & $\begin{array}{l}8.8 \text { vs } 6.3(\mathrm{HR}, 0.55 ; 95 \% \mathrm{Cl} \text {, } \\
0.40-0.77, P=0.00016)\end{array}$ \\
\hline
\end{tabular}

Abbreviations: $\mathrm{mCRC}$, metastatic colorectal cancer; PFS, progression-free survival; OS, overall survival; Cl, confidence interval; HR, hazard ratio; $\mathrm{NR}$, not reported.

with heavily pretreated $\mathrm{CRC}$, including anti-EGFR antibody in KRAS wt patients (Table 3). One partial response and 19 stable diseases were seen among 27 evaluable patients. Median PFS was 107 days (95\% CI, 66-161). The most commonly seen treatment-related toxicity included hand-foot skin reaction, fatigue, and rash. ${ }^{57,58}$

The CORRECT trial, a multinational, multicenter, randomized, placebo-controlled, Phase 3 trial was conducted based on the Phase $1 / 1 \mathrm{~b}$ safety and efficacy data, with a primary endpoint of OS. ${ }^{59} \mathrm{~A}$ total of 760 patients were randomized to either regorafenib or placebo in a 2:1 ratio. Improved median OS was observed in the regorafenib group compared to the placebo group, 6.4 months vs 5.0 months, respectively (HR, 0.77; 95\% CI, 0.64-0.94, one-sided $P=0.0052$ ).

This benefit of regorafenib in OS was also demonstrated in a second multinational multicenter randomized Phase 3 trial, CONCUR trial, conducted in 204 Asian patients. ${ }^{60}$ Patients were randomized to either regorafenib or placebo in a 2:1 ratio. After a median follow-up of 7.4 months, a significant prolonged OS was observed associated with regorafenib treatment compared to placebo (HR, 0.55; $95 \%$ CI, 0.44-0.77, one-sided $P=0.00016$ ); with median OS of 8.8 months in the regorafenib-treated group vs 6.3 months in the placebo group.

Interestingly, in the CORRECT trial, although no difference in median PFS between the regorafenib or placebo group (1.9 vs 1.7 months, respectively) was seen, a PFS HR of 0.49 (95\% CI, 0.42-0.581, $P<0.0001$ ), adjusted for stratification factors, was highly statistically significant, indicating a $51 \%$ reduction in the hazard of disease progression or death for patients receiving regorafenib. The Kaplan-Meier curves diverged after around $50 \%$ of the patients had progressed at the first scan obtained at 8 weeks. ${ }^{59} \mathrm{~A}$ similar observation was made in the CONCUR trial, ${ }^{60}$ suggesting a refined subgroup of patients is likely to benefit from such therapy.

Despite the statistically significant benefit of regorafenib observed in Phase 3 clinical trials, a substantial group of patients failed to respond to or continue to progress with the treatment. The toxicity profile can also make treatment with regorafenib challenging, in particular in the palliative salvage therapy setting. Therefore, further identification and validation of potential molecular markers might help to refine the patient population that will most likely benefit from regorafenib and to avoid unnecessary adverse effects in patients who are not likely to respond.

\section{Molecular markers in oncogenic pathways}

RAS/RAF/MAPK and PI3K/AKT signaling pathways are essential in EGFR-mediated CRC oncogenesis. Multiple biomarkers identified in these pathways have improved the success of other targeted therapies, and their associations with regorafenib response have been recently investigated.

In the CORRECT trail, $57 \%$ of patients harbored a KRAS mutation; however, $K R A S$ status was not predictive of OS or PFS associated with regorafenib. ${ }^{59}$ Seventy percent of patients were assessed for $K R A S$ status, and $21 \%$ were analyzed for $B R A F$ mutation status in the CONCUR trial. Consistent with the findings in the CORRECT trial, neither $K R A S$ nor $B R A F$ was predictive for a response to regorafenib. ${ }^{60}$

Further biomarker analysis on CORRECT trial data also confirmed that regorafenib provided clinical benefits in various mutational subgroups. ${ }^{61}$ Correlative analysis based on mutational status by BEAMing ${ }^{62}$ of circulating plasma DNA demonstrated regorafenib benefit across patient groups defined by KRAS and PIK3CA mutational status, and that no significant difference was revealed between wt and mutant tumors for either $K R A S$ or PIK3CA, consistent with additional anti-angiogenesis activity of regorafenib. ${ }^{63}$ The correlation between the circulating DNA level and clinical outcome was also assessed in the same study. Although a high circulating DNA level does not predict regorafenib clinical response, it is associated with shorter OS than that in patients with a lower baseline circulating DNA level regardless of treatment.

Gene expression profile (eg, chromosomal instability, deficient MMR, KRAS mutant) was obtained in tumors from 
CORRECT trial by next generation sequencing in a recent study. The benefit of regorafenib in PFS was observed in patients with high-risk molecular characteristics $(\mathrm{HR}=0.10$; 95\% CI $0.02-0.35, P=0.0009)$ more than in the low-risk subgroup (HR $=0.58 ; 95 \%$ CI $0.44-0.77, P=0.002)$, indicating a role of molecular stratification, including chromosome instability, in identifying the appropriate patient population for regorafenib treatment. ${ }^{64}$

\section{Molecular biomarkers in angiogenesis pathway}

Angiogenesis is an essential component and a rate-limiting step of tumor growth and metastasis in CRC. ${ }^{65}$ It is a complex network of pathways controlled by naturally-occurring growth factors, including VEGF, FGF, and PDGF system. It is also regulated by HIF-1, a transcription factor that induces VEGF expression. Anti-angiogenic therapies, such as bevacizumab, sorafenib, and sunitinib, have shown benefit in various solid tumors, including mCRC. Regorafenib is among the small molecule kinase inhibitors with anti-angiogenic activity.

The identification and validation of molecular markers for successful angiogenesis inhibition is an ongoing challenge, with most of the evidence based on bevacizumab treatment in the pre-regorafenib era. Despite extensive research, no validated angiogenesis biomarkers have yet been established. For example, studies have failed to show the predictive value of tumor VEGF expression levels or baseline circulating VEGF level in bevacizumab treatment response. ${ }^{66-69}$

The association between regorafenib response and the level of plasma biomarkers involved in angiogenesis has been previously reported in a small clinical study. ${ }^{70}$ Increased VEGF-family ligands concentration (eg, PIGF), and decreased TIMP2 and soluble VEGF receptor 2 and TIE1 levels, were detected in response to regorafenib. Higher baseline TIMP2 and soluble TIE1 were correlated with tumor shrinkage with regorafenib treatment, while higher baseline concentration of angiopoietin and soluble TIE2, a crucial regulator of angiogenesis, seemed to be associated with an increased risk of progression. ${ }^{70}$

Angiogenesis biomarkers were also analyzed in correlative studies embedded in the CORRECT trial. Higher levels of soluble protein TIE-1 were found to be associated with higher regorafenib benefit in OS, consistent with previous observations (Tabernero et al manuscript in press in Lancet Oncology, 2015). Also consistent with previous findings, ${ }^{71,72}$ an association between high IL-8 and PIGF levels and poor clinical outcomes was detected in the placebo-treated mCRC patients, suggesting a prognostic value of these plasma proteins. These findings will, however, need to be validated in further studies.

Genotyping in $V E G F$ and $V E G F R$ was also used to identify single nucleotide polymorphisms to determine regorafenib treatment outcome in mCRC. In a recent study, 138 samples from $\mathrm{mCRC}$ patients were tested for single nucleotide polymorphisms in $V E G F-A, V E G F-C$, and $V E G F R 1-3,{ }^{73}$ and $V E G F-A$ rs2010963 showed a correlation with PFS and OS, with HR of 0.49 (95\% CI, 0.33-0.81) and 0.52 (95\% CI, 0.34-0.99), respectively, indicating its use as patient selection tool for consideration for regorafenib treatment.

\section{Future directions}

The understanding of the molecular profile and the identification of biomarkers associated with targeted therapies' outcomes is crucial for the success of personalized medicine. Despite ongoing investigation, no validated biomarkers are currently available to guide the use of regorafenib; therefore, further research efforts are essential to delineate its role in the treatment of mCRC.

Regorafenib is currently used as a treatment option in $\mathrm{mCRC}$ after progression on other standard therapies, a disease with high biologic heterogeneity. Given the multiple kinase inhibition profile of regorafenib, a full understanding of the complex network of pathways, especially the cross-talk and overlapping of angiogenesis and oncogenesis pathways, is critical for the continued search for meaningful molecular markers. It is very likely that a biomarker signature, rather than a single marker, will predict the activity of regorafenib in individual patients. The potential molecular markers might also need to be evaluated in combination with biomarkers involving other biological pathways in order to demonstrate the abilities to predict clinical outcomes.

The lack of available angiogenesis biomarkers also makes molecular profiling of $\mathrm{mCRC}$ treated with regorafenib more challenging. Multiple potential molecular markers in angiogenesis were evaluated in previous studies, including circulating soluble KIT levels as potential markers for sunitinib treatment in gastrointestinal stromal tumors (GIST), ${ }^{74}$ CD31 and PDGFR-A in bevacizumab treated breast cancer, ${ }^{75}$ cytokine TNF- $\alpha$ in sunitinib treated renal cell carcinoma, ${ }^{76}$ and polymorphisms in IL-8 allele in bevacizumab treated ovarian cancer, ${ }^{77}$ which will benefit our future research efforts. In the era of next generation sequencing, extended genetic analysis will also facilitate our understanding of the genetic landscape of mCRC.

One of the main challenges of targeted therapy is secondary resistance to treatments. Biomarker-guided patient 
selection might help to increase the success rate of such treatments. However, a substantial group of patients eventually develop secondary therapy resistance and progress, which reflects additional molecular and genetic modifications of tumors, suggesting a role of further, longitudinal biomarker monitoring and the identification of secondary biomarkers to guide the next line of treatment. This is of high importance for regorafenib treatment since most patients conceivably have already developed resistance towards other previous targeted therapies and the majority of patients who initially respond to regorafenib later show disease progression. Reevaluation of the molecular profiling upon secondary treatment failure might identify potential therapeutic targets with potential to improve the outcomes on regorafenib.

Understanding the treatment of $\mathrm{mCRC}$ at the molecular level is an actively growing field. Treatment outcomes have been significantly improved over the last decade with the availability of biomarkers associated with treatment response. Identification of new robust molecular markers based on our knowledge of mCRC biology will further improve survival outcome through accurate patient selection, not only in regorafenib treatment, but also in other novel therapies for $\mathrm{mCRC}$.

\section{Disclosure}

The Mayo Clinic Foundation received grant funding from Bayer Oncology for research led by author AG. The authors report no other conflicts of interest in this work.

\section{References}

1. American Cancer Society. Cancer Facts. Available from: http://www. cancer.org/acs/groups/content/@editorial/documents/document/acspc044552.pdf. American Cancer Society; 2015. Accessed September 13, 2015.

2. Dahabreh IJ, Terasawa T, Castaldi PJ, Trikalinos TA. Systematic review: Anti-epidermal growth factor receptor treatment effect modification by KRAS mutations in advanced colorectal cancer. Ann Intern Med. 2011;154(1):37-49.

3. Fernandez-Medarde A, Santos E. Ras in cancer and developmental diseases. Genes Cancer. 2011;2(3):344-358.

4. Peeters M, Oliner KS, Parker A, et al. Massively parallel tumor multigene sequencing to evaluate response to panitumumab in a randomized phase III study of metastatic colorectal cancer. Clin Cancer Res. 2013; 19(7):1902-1912.

5. Douillard JY, Oliner KS, Siena S, et al. Panitumumab-FOLFOX4 treatment and RAS mutations in colorectal cancer. N Engl J Med. 2013;369(11): 1023-1034.

6. Douillard JY, Siena S, Cassidy J, et al. Final results from PRIME: randomized phase III study of panitumumab with FOLFOX4 for firstline treatment of metastatic colorectal cancer. Ann Oncol. 2014;25(7): $1346-1355$.

7. Van Cutsem E, Peeters M, Siena S, et al. Open-label phase III trial of panitumumab plus best supportive care compared with best supportive care alone in patients with chemotherapy-refractory metastatic colorectal cancer. J Clin Oncol. 2007;25(13):1658-1664.
8. Andreyev HJ, Norman AR, Cunningham D, et al. Kirsten ras mutations in patients with colorectal cancer: the 'RASCAL II' study. Br J Cancer. 2001;85(5):692-696.

9. Esteller M, Gonzalez S, Risques RA, et al. K-ras and p16 aberrations confer poor prognosis in human colorectal cancer. J Clin Oncol. 2001; 19(2):299-304.

10. Amado RG, Wolf M, Peeters M, et al. Wild-type KRAS is required for panitumumab efficacy in patients with metastatic colorectal cancer. J Clin Oncol. 2008;26(10):1626-1634.

11. Price TJ, Peeters M, Kim TW, et al. Panitumumab versus cetuximab in patients with chemotherapy-refractory wild-type KRAS exon 2 metastatic colorectal cancer (ASPECCT): a randomised, multicentre, open-label, non-inferiority phase 3 study. Lancet Oncol. 2014;15(6): $569-579$.

12. Jonker DJ, O'Callaghan CJ, Karapetis CS, et al. Cetuximab for the treatment of colorectal cancer. N Engl J Med. 2007;357(20):2040-2048.

13. Karapetis CS, Khambata-Ford S, Jonker DJ, et al. K-ras mutations and benefit from cetuximab in advanced colorectal cancer. $N$ Engl J Med. 2008;359(17):1757-1765.

14. Van Cutsem E, Kohne CH, Hitre E, et al. Cetuximab and chemotherapy as initial treatment for metastatic colorectal cancer. $N$ Engl J Med. 2009;360(14):1408-1417.

15. Van Cutsem E, Kohne CH, Lang I, et al. Cetuximab plus irinotecan, fluorouracil, and leucovorin as first-line treatment for metastatic colorectal cancer: updated analysis of overall survival according to tumor KRAS and BRAF mutation status. J Clin Oncol. 2011;29(15):2011-2019.

16. NCCN. Guidelines for Colon Cancer Version 3. Available from: http:// www.nccn.org/professionals/physician_gls/pdf/colon.pdf. NCCN; 2015. Accessed September 13, 2015.

17. Bokemeyer C, Van Cutsem E, Rougier P, et al. Addition of cetuximab to chemotherapy as first-line treatment for KRAS wild-type metastatic colorectal cancer: pooled analysis of the CRYSTAL and OPUS randomised clinical trials. Eur J Cancer. 2012;48(10):1466-1475.

18. Tran B, Kopetz S, Tie J, et al. Impact of BRAF mutation and microsatellite instability on the pattern of metastatic spread and prognosis in metastatic colorectal cancer. Cancer. 2011;117(20):4623-4632.

19. Joyce T, Oikonomou E, Kosmidou V, et al. A molecular signature for oncogenic BRAF in human colon cancer cells is revealed by microarray analysis. Curr Cancer Drug Targets. 2012;12(7):873-898.

20. Tol J, Nagtegaal ID, Punt CJ. BRAF mutation in metastatic colorectal cancer. N Engl J Med. 2009;361(1):98-99.

21. Richman SD, Seymour MT, Chambers P, et al. KRAS and BRAF mutations in advanced colorectal cancer are associated with poor prognosis but do not preclude benefit from oxaliplatin or irinotecan: results from the MRC FOCUS trial. J Clin Oncol. 2009;27(35):5931-5937.

22. Loupakis F, Cremolini C, Masi G, et al. Initial therapy with FOLFOXIRI and bevacizumab for metastatic colorectal cancer. N Engl J Med. 2014;371(17):1609-1618.

23. Loupakis F, Cremolini C, Salvatore L, et al. FOLFOXIRI plus bevacizumab as first-line treatment in BRAF mutant metastatic colorectal cancer. Eur J Cancer. 2014;50(1):57-63.

24. Yuan ZX, Wang XY, Qin QY, et al. The prognostic role of BRAF mutation in metastatic colorectal cancer receiving anti-EGFR monoclonal antibodies: a meta-analysis. PLoS One. 2013;8(6):e65995.

25. Pietrantonio F, Petrelli F, Coinu A, et al. Predictive role of BRAF mutations in patients with advanced colorectal cancer receiving cetuximab and panitumumab: a meta-analysis. Eur J Cancer. 2015;51(5):587-594.

26. Kopetz S, Desai J, Chan E, et al. PLX4032 in metastatic colorectal cancer patients with mutant BRAF tumors. Journal of Clinical Oncology. 2010;28(15)

27. Prahallad A, Sun C, Huang S, et al. Unresponsiveness of colon cancer to BRAF(V600E) inhibition through feedback activation of EGFR. Nature. 2012;483(7387):100-103.

28. Corcoran RB, Ebi H, Turke AB, et al. EGFR-mediated re-activation of MAPK signaling contributes to insensitivity of BRAF mutant colorectal cancers to RAF inhibition with vemurafenib. Cancer Discov. 2012;2(3):227-235. 
29. Oikonomou E, Koc M, Sourkova V, Andera L, Pintzas A. Selective BRAFV600E inhibitor PLX4720, requires TRAIL assistance to overcome oncogenic PIK3CA resistance. PLoS One. 2011;6(6):e21632.

30. Yang H, Higgins B, Kolinsky K, et al. Antitumor activity of BRAF inhibitor vemurafenib in preclinical models of BRAF-mutant colorectal cancer. Cancer Res. 2012;72(3):779-789.

31. Corcoran RB, Falchook GS, Infante JR, et al. BRAF V600 mutant colorectal cancer (CRC) expansion cohort from the phase I/II clinical trial of BRAF inhibitor dabrafenib (GSK2118436) plus MEK inhibitor trametinib (GSK1120212). Journal of Clinical Oncology. 2012;30(15).

32. Yaeger R, Cercek A, O'Reilly EM, et al. Pilot trial of combined BRAF and EGFR inhibition in BRAF-mutant metastatic colorectal cancer patients. Clin Cancer Res. 2015;21(6):1313-1320.

33. Tabernero J, Chan E, Baselga J, et al. VE-BASKET, a Simon 2-stage adaptive design, phase II, histology-independent study in nonmelanoma solid tumors harboring BRAF V600 mutations (V600m): Activity of vemurafenib (VEM) with or without cetuximab (CTX) in colorectal cancer (CRC). ASCO Meeting Abstracts. 2014;32(15 suppl):3518.

34. Atreya CE, Van Cutsem E, Bendell JC, et al. Updated efficacy of the MEK inhibitor trametinib (T), BRAF inhibitor dabrafenib (D), and anti-EGFR antibody panitumumab $(\mathrm{P})$ in patients (pts) with BRAF V600E mutated (BRAFm) metastatic colorectal cancer (mCRC). ASCO Meeting Abstracts. 2015;33(15 suppl):103.

35. Tabernero J, van Geel R, Bendell JC, et al. Phase I study of the selective BRAFV600 inhibitor encorafenib (LGX818) combined with cetuximab and with or without the a-specific PI3K inhibitor alpelisib (BYL719) in patients with advanced BRAF mutant colorectal cancer. Eur J Cancer. 2014;50:199-199.

36. Hong DS, Morris VK, El Osta BE, et al. Phase Ib study of vemurafenib in combination with irinotecan and cetuximab in patients with BRAFmutated metastatic colorectal cancer and advanced cancers. ASCO Meeting Abstracts. 2015;33(15 suppl):3511.

37. Ishida Y, Agata Y, Shibahara K, Honjo T. Induced expression of PD-1, a novel member of the immunoglobulin gene superfamily, upon programmed cell death. EMBO J. 1992;11(11):3887-3895.

38. Nishimura H, Okazaki T, Tanaka Y, et al. Autoimmune dilated cardiomyopathy in PD-1 receptor-deficient mice. Science. 2001;291(5502): 319-322.

39. Dong H, Strome SE, Salomao DR, etal. Tumor-associated B7-H1 promotes T-cell apoptosis: a potential mechanism of immune evasion. Nat Med. 2002;8(8):793-800.

40. Herbst RS, Soria JC, Kowanetz M, et al. Predictive correlates of response to the anti-PD-L1 antibody MPDL3280A in cancer patients. Nature. 2014;515(7528):563-567.

41. Powles T, Eder JP, Fine GD, et al. MPDL3280A (anti-PD-L1) treatment leads to clinical activity in metastatic bladder cancer. Nature. 2014; 515(7528):558-562.

42. Brahmer JR, Tykodi SS, Chow LQ, et al. Safety and activity of antiPD-L1 antibody in patients with advanced cancer. N Engl J Med. 2012; 366(26):2455-2465.

43. Topalian SL, Hodi FS, Brahmer JR, et al. Safety, activity, and immune correlates of anti-PD-1 antibody in cancer. $N$ Engl J Med. 2012; 366(26):2443-2454.

44. Garon EB, Rizvi NA, Hui R, et al. Pembrolizumab for the treatment of non-small-cell lung cancer. $N$ Engl J Med. 2015;372(21): 2018-2028.

45. Brahmer J, Reckamp KL, Baas P, et al. Nivolumab versus Docetaxel in Advanced Squamous-Cell Non-Small-Cell Lung Cancer. $N$ Engl J Med. 2015;373(2):123-135.

46. Brahmer JR, Drake CG, Wollner I, et al. Phase I study of single-agent anti-programmed death-1 (MDX-1106) in refractory solid tumors: safety, clinical activity, pharmacodynamics, and immunologic correlates. J Clin Oncol. 2010;28(19):3167-3175.

47. Tumeh PC, Harview CL, Yearley JH, et al. PD-1 blockade induces responses by inhibiting adaptive immune resistance. Nature. 2014; 515(7528):568-571.
48. Gros A, Robbins PF, Yao X, et al. PD-1 identifies the patientspecific $\mathrm{CD} 8(+)$ tumor-reactive repertoire infiltrating human tumors. J Clin Invest. 2014;124(5):2246-2259.

49. Timmermann B, Kerick M, Roehr C, et al. Somatic mutation profiles of MSI and MSS colorectal cancer identified by whole exome next generation sequencing and bioinformatics analysis. PLoS One. 2010; 5(12):e15661.

50. Dolcetti R, Viel A, Doglioni C, et al. High prevalence of activated intraepithelial cytotoxic $\mathrm{T}$ lymphocytes and increased neoplastic cell apoptosis in colorectal carcinomas with microsatellite instability. $\mathrm{Am}$ J Pathol. 1999;154(6):1805-1813.

51. Lipson EJ, Sharfman WH, Drake CG, et al. Durable cancer regression off-treatment and effective reinduction therapy with an anti-PD-1 antibody. Clin Cancer Res. 2013;19(2):462-468.

52. Le DT, Uram JN, Wang H, et al. PD-1 Blockade in Tumors with MismatchRepair Deficiency. N Engl J Med. 2015;372(26):2509-2520.

53. Le DT, Uram JN, Wang H, et al. PD-1 blockade in tumors with mismatch repair deficiency. ASCO Meeting Abstracts. 2015;33(18 suppl): LBA100.

54. Stivarga ${ }^{\circledR}$ (regorafenib) tablets, oral [prescribing information]. Wayne, NJ: Bayer HealthCare Pharmaceuticals Inc.; 2012.

55. Wilhelm SM, Dumas J, Adnane L, et al. Regorafenib (BAY 73-4506): a new oral multikinase inhibitor of angiogenic, stromal and oncogenic receptor tyrosine kinases with potent preclinical antitumor activity. Int J Cancer. 2011;129(1):245-255.

56. Abou-Elkacem L, Arns S, Brix G, et al. Regorafenib inhibits growth, angiogenesis, and metastasis in a highly aggressive, orthotopic colon cancer model. Mol Cancer Ther. 2013;12(7):1322-1331.

57. Strumberg D, Scheulen ME, Schultheis B, et al. Regorafenib (BAY 73-4506) in advanced colorectal cancer: a phase I study. Br J Cancer. 2012;106(11):1722-1727

58. Mross K, Frost A, Steinbild S, et al. A phase I dose-escalation study of regorafenib (BAY 73-4506), an inhibitor of oncogenic, angiogenic, and stromal kinases, in patients with advanced solid tumors. Clin Cancer Res. 2012;18(9):2658-2667.

59. Grothey A, Van Cutsem E, Sobrero A, et al. Regorafenib monotherapy for previously treated metastatic colorectal cancer (CORRECT): an international, multicentre, randomised, placebo-controlled, phase 3 trial. Lancet. 2013;381(9863):303-312.

60. Li J, Qin S, Xu R, et al. Regorafenib plus best supportive care versus placebo plus best supportive care in Asian patients with previously treated metastatic colorectal cancer (CONCUR): a randomised, double-blind, placebo-controlled, phase 3 trial. Lancet Oncol. 2015; 16(6):619-629.

61. Jeffers M, Van Cutsem E, Sobrero AF, et al. Mutational analysis of biomarker samples from the CORRECT study: Correlating mutation status with clinical response to regorafenib. J Clin Oncol. 2013;(suppl 4):381.

62. Diehl F, Li M, He Y, Kinzler KW, Vogelstein B, Dressman D. BEAMing: single-molecule PCR on microparticles in water-in-oil emulsions. Nat Methods. 2006;3(7):551-559.

63. Tabernero J, Lenz HJ, Siena S, et al. Analysis of circulating DNA and protein biomarkers to predict the clinical activity of regorafenib and assess prognosis in patients with metastatic colorectal cancer: a retrospective, exploratory analysis of the CORRECT trial. Lancet Oncol. 2015;16(8):937-948.

64. Teufel M, Schwenke S, Seidel H, et al. Molecular subtypes and outcomes in regorafenib-treated patients with metastatic colorectal cancer (mCRC) enrolled in the CORRECT trial. ASCO Meeting Abstracts. 2015;33(15 suppl):3558.

65. Carmeliet P, Jain RK. Angiogenesis in cancer and other diseases. Nature. 2000;407(6801):249-257.

66. Willett CG, Duda DG, di Tomaso E, et al. Efficacy, safety, and biomarkers of neoadjuvant bevacizumab, radiation therapy, and fluorouracil in rectal cancer: a multidisciplinary phase II study. J Clin Oncol. 2009; 27(18):3020-3026. 
67. Kopetz S, Hoff PM, Morris JS, et al. Phase II trial of infusional fluorouracil, irinotecan, and bevacizumab for metastatic colorectal cancer: efficacy and circulating angiogenic biomarkers associated with therapeutic resistance. J Clin Oncol. 2010;28(3):453-459.

68. Brostjan C, Gebhardt K, Gruenberger B, et al. Neoadjuvant treatment of colorectal cancer with bevacizumab: the perioperative angiogenic balance is sensitive to systemic thrombospondin-1 levels. Clin Cancer Res. 2008;14(7):2065-2074.

69. Jubb AM, Hurwitz HI, Bai W, et al. Impact of vascular endothelial growth factor-A expression, thrombospondin-2 expression, and microvessel density on the treatment effect of bevacizumab in metastatic colorectal cancer. J Clin Oncol. 2006;24(2):217-227.

70. Eisen T, Joensuu H, Nathan PD, et al. Regorafenib for patients with previously untreated metastatic or unresectable renal-cell carcinoma: a single-group phase 2 trial. Lancet Oncol. 2012;13(10): 1055-1062.

71. Sung CY, Son MW, Ahn TS, Jung DJ, Lee MS, Baek MJ. Expression of placenta growth factor in colorectal carcinomas. J Korean Soc Coloproctol. 2012;28(6):315-320.

72. Ning Y, Lenz HJ. Targeting IL-8 in colorectal cancer. Expert Opin Ther Targets. 2012;16(5):491-497.
73. Giampieri R, Salvatore L, Del Prete M, et al. Angiogenesis genotyping and clinical outcome during regorafenib treatment in metastatic colorectal cancer patients. ASCO Meeting Abstracts. 2015;33(3 suppl):595.

74. Deprimo SE, Huang X, Blackstein ME, et al. Circulating levels of soluble KIT serve as a biomarker for clinical outcome in gastrointestinal stromal tumor patients receiving sunitinib following imatinib failure. Clin Cancer Res. 2009;15(18):5869-5877.

75. Yang SX, Steinberg SM, Nguyen D, Wu TD, Modrusan Z, Swain SM. Gene expression profile and angiogenic marker correlates with response to neoadjuvant bevacizumab followed by bevacizumab plus chemotherapy in breast cancer. Clin Cancer Res. 2008;14(18):5893-5899.

76. Perez-Gracia JL, Prior C, Guillen-Grima F, et al. Identification of TNFalpha and MMP-9 as potential baseline predictive serum markers of sunitinib activity in patients with renal cell carcinoma using a human cytokine array. Br J Cancer. 2009;101(11):1876-1883.

77. Schultheis AM, Lurje G, Rhodes KE, et al. Polymorphisms and clinical outcome in recurrent ovarian cancer treated with cyclophosphamide and bevacizumab. Clin Cancer Res. 2008;14(22):7554-7563.

78. Falchook GS, Long GV, Kurzrock R, et al. Dabrafenib in patients with melanoma, untreated brain metastases, and other solid tumours a phase 1 dose-escalation trial. Lancet. 2012;379(9829):1893-1901.
OncoTargets and Therapy

\section{Publish your work in this journal}

OncoTargets and Therapy is an international, peer-reviewed, open access journal focusing on the pathological basis of all cancers, potential targets for therapy and treatment protocols employed to improve the management of cancer patients. The journal also focuses on the impact of management programs and new therapeutic agents and protocols on

\section{Dovepress}

patient perspectives such as quality of life, adherence and satisfaction. The manuscript management system is completely online and includes a very quick and fair peer-review system, which is all easy to use. Visit http://www.dovepress.com/testimonials.php to read real quotes from published authors. 